JOURNAL OF INTEGRAL EQUATIONS

AND APPLICATIONS

Volume 5, Number 1, Winter 1993

\title{
OPEN PROBLEMS IN SINGULAR INTEGRAL THEORY
}

\author{
WILLIAM R. DERRICK
}

\begin{abstract}
The following list of 19 problems were proposed by participants of the singular integral operators NSFCBMS regional research conference, held at the University of Montana from August 26-September 1, 1989. The contributing participant is noted in parentheses at the beginning of each problem. A number of other open problems appear in the CBMS monograph Singular Integral Operators (to appear) detailing the contents of the lectures presented by Professor F. Michael Christ during this conference. It is hoped that the monograph and these open problems will serve to inspire continued work in this fascinating and useful area of mathematics. Symbols and concepts not defined in these problems are explained in the monograph or in the two books listed in the references.
\end{abstract}

\section{Open problems.}

1. (J. Alvarez). Consider the class $\mathcal{M}$ of operators $K$ acting on Schwartz functions:

$$
K f=\int e^{-2 \pi i x \circ \xi} \rho(x, \xi) \hat{f}(\xi) d \xi+R f
$$

where

$$
\left|D^{\alpha} x D_{\xi}^{\beta} \rho(x, \xi)\right| \leq C_{\alpha, \beta}(1+|\xi|)^{-|\beta|+\delta|\alpha|},
$$

$0<\delta<1,|\alpha| \leq M,|\beta| \leq N$, for some $M, N$, and

$$
R, R \frac{\partial}{\partial x_{j}}, \frac{\partial}{\partial x_{j}} R, \quad 1 \leq j \leq n, \quad \text { map } L^{p} \text { into itself, }
$$

for $p_{0} \leq p \leq p_{0}^{\prime}$, for some $1<p_{0} \leq 2$. Then $\mathcal{M}$ is a self-adjoint Banach algebra with the norm

$$
\begin{aligned}
& \|K\|_{\mathcal{M}}=\inf \left\{\inf _{|\alpha| \leq M,|\beta| \leq N} C_{\alpha, \beta}+\|R\|_{L^{p_{0}}}+\|R\|_{L^{p_{0}^{\prime}}}\right. \\
& \left.+\sum_{j=1}^{n}\left(\left\|\frac{\partial}{\partial x_{j}} R\right\|_{L^{p_{0}}}+\left\|R \frac{\partial}{\partial x_{j}}\right\|_{L^{p_{0}}}+\left\|\frac{\partial}{\partial x_{j}} R\right\|_{L^{p_{0}^{\prime}}}+\left\|R \frac{\partial}{\partial x_{j}}\right\|_{L^{p_{0}^{\prime}}}\right)\right\},
\end{aligned}
$$

Received by the editors on July 21, 1992.

Copyright (C)1993 Rocky Mountain Mathematics Consortium 
where the infimum is taken over all possible representations of $K$ as (1). Take $K$ in $\mathcal{M}$, and suppose $K$ is a self-adjoint operator on $L^{2}$. Question: Is it possible to find $C>0, \mu \in \mathbf{R}$, such that

$$
\left\|e^{2 \pi i t K}\right\|_{\mathcal{M}} \leq C(1+|t|)^{\mu}, \quad t \in \mathbf{R} ?
$$

A positive answer to this question implies the existence of a functional calculus on $\mathcal{M}$, defined by means of the $\mathrm{H}$. Weyl formula

$$
f(K)=\int_{-\infty}^{\infty} e^{-2 \pi i t K} \hat{f}(t) d t
$$

for a class of functions $f$ with a finite number of derivatives. The question has a positive answer when $p_{0}=2$ or when $\mathcal{M}$ is defined on a compact manifold without boundary of class $C^{r}$, for some $r=$ $r(M, N)<\infty$. Certainly the class $\mathcal{M}$ can be modified adding more terms in the first term in (1), in order to get smoother error $R$. One of the nice features of (2) is to admit an immediate extension to $n$-tuples of non necessarily commuting operators. [Note: See "Functional calculi of pseudo-differential operators, III," to appear in Studia Mathematica.]

2. (C.J. Bishop and P.W. Jones). Suppose $\Gamma$ is a Lipschitz graph and $E \subset \Gamma$ satisfies $\Lambda_{1}(E)>0$. Using the boundedness of the Cauchy integral on $L^{2}(\Gamma, d s)$ and a duality argument, it is shown that this implies $\gamma(E)>0$, i.e., there exist nonconstant holomorphic functions on $\mathbf{C} \backslash E$. Given $\Gamma$ and $E$ construct such a function. In other words, prove the Denjoy conjecture without using duality.

3. (G. David). Let $\Gamma$ be a chord-arc curve, with constant $\leq C$, contained in $\mathbf{R}^{2}$, going through 0 . (This means that if $x, y \in \Gamma$, the arc joining $x$ to $y$ has length $\leq C|x-y|$.) Given $\varepsilon>0$, does there exist $\eta>0$, that depends only on $\varepsilon$ and $C$, such that if $E \subset \Gamma$ has length $\geq \varepsilon$ and is contained in $B(0,1)$, then there is a line $L$ such that $\left|\prod_{L}(E)\right| \geq \eta$, where $\prod_{L}$ is the orthogonal projection onto $L$. If the answer is yes, do this for regular curves, and then do it in higher dimensions. [Note: the order of the quantifiers is important.]

4. (J. Garnett). Let $K$ be a compact plane set and let $T$ be an affine map, not complex affine. 
(a) If $K$ has positive analytic capacity, does $T(K)$ ? (Only interesting if it has dimension 1).

(b) Assume $K$ has dimension 1 and is Ahlfors-David (AD) regular. If the Cauchy integral is bounded on $\left(K, \Lambda_{1}\right)$, is it bounded on $\left(T(K), \Lambda_{1}\right)$ ?

5. (J. Garnett). Let $K$ be a compact plane set such that $0<\Lambda_{1}(K)<$ $\infty$. Assume $K$ is $A D$ regular but Besicovitch irregular. Prove that the analytic capacity of $K, \gamma(K)=0$.

6. (J. Garnett). Let $K$ be a compact plane set such that $\gamma(K)>0$. Prove or disprove: There exists a finite Borel measure $\mu$ supported on $K$ such that

$$
F(z)=\int \frac{d \mu(w)}{w-z}
$$

is bounded and nonconstant on $K^{C}$. [Note: This may be false].

7. (D. Hamilton). Find necessary and sufficient conditions on a rectifiable curve $\gamma \subset \mathbf{R}^{2}$ so that the Fredholm-Poincaré operator is a bounded $L^{2}$ operator.

8. (D. Hamilton). Ahlfors has shown that singular integral operators may be used to characterize quasiconformal deformations in $\mathbf{R}^{n}$. Develop a nonlocal theory for general quasiconformal maps.

9. (M. Jodeit). For which singular measure $\mu$ on $\mathbf{R}$ is $H \mu$ Denjoy integrable on almost all intervals $(a, b)$ ?

10. (M. Jodeit). Which functions $h(x)$ defined on $\mathbf{R}$ are Hilbert transforms of integrable functions? [Necessary conditions: $h \in L^{1, \infty}$ (weak $L^{1}$ ), and

$$
\lim _{m \rightarrow \infty} \int_{a}^{b} h_{m}(x) d x \quad \text { exists, for almost all }(a, b),
$$

where

$$
h_{m}(x)= \begin{cases}h(x), & |h(x)| \leq m, \\ m \operatorname{sgn} h(x), & \text { otherwise }\end{cases}
$$


11. (M. Jodeit). Answer this question as above for the Riesz system on $\mathbf{R}^{n}$.

12. (M. Jodeit). Is the characteristic function of the perfect squares a (Fourier series) multiplier of type $(p, p)$ for some $p \neq 2$ ? In particular, is it of weak type $(2,4)$ ? [Z. Zalcwasser (Studia Math. 7 (1938), 16-35) shows it cannot be of type $(2,4)$.]

13. (T. Trent). Let $P^{2}(\mu)$ denote the closure of the analytic polynomials in $L^{2}(\mu)$. Fix a compact set $K$ in $\mathbf{C}$. It is known that either $P^{2}\left(\left.d A\right|_{K}\right)=L^{2}\left(\left.d A\right|_{K}\right)$ or else $P^{2}\left(\left.d A\right|_{K}\right)$ has analytic bounded point elevations. Find geometric or quasi-geometric conditions on $K$ to characterize when $P^{2}\left(\left.d A\right|_{K}\right) \neq L^{2}\left(\left.d A\right|_{K}\right)$.

14. (T. Trent). A bounded set $E$ in $\mathbf{C}$ is of finite perimeter if there exists a complex Borel measure $\nu$ such that $\hat{\nu}=\chi_{E}$ a.e.-area Lebesgue measure on $\mathbf{C}$. Here $\hat{\nu}$ denotes the Cauchy transform of $\nu$. Does there exist a compact connected set $K$ such that $R(K) \neq C(K)$ and $K$ does not contain a (nontrivial) set of finite perimeter?

15. (G. Verchota). Let $\partial D$ be a Lipschitz graph in $\mathbf{R}^{n}$,

$$
K f(P)=\operatorname{p.v} \cdot \frac{1}{\omega_{n}} \int_{\partial D} \frac{N_{Q} \circ(Q-P)}{|Q-P|^{n}} f(Q) d Q
$$

where $\omega_{n}$ is the surface area of $S^{n-1}, d Q$ is the Lebesgue surface measure, $N_{Q}=\left(N_{Q}^{1}, N_{Q}^{2}, \ldots, N_{Q}^{n}\right)$ is the unit normal vector at $Q \in \partial D$, $\pm(1 / 2) I+K$ are the traces from inside and outside $D \subset \mathbf{R}^{n}$ of the classical double layer potential. If $\lambda$ is in the spectrum of $K$, is $|\lambda|<1 / 2$ ? [Note: The answer is yes for $C^{1}$ and small Lipschitz norms.]

16. (G. Verchota). Let $\Delta \vec{u}+\nabla \operatorname{div} \vec{u}=\overrightarrow{0}$ in $D \subset \mathbf{R}^{n}$, (a system with $\left.\vec{u}=\left(u_{1}, u_{2}, \ldots, u_{n}\right)\right)$, and suppose on the boundary that

$$
\sum_{j=1}^{n}\left(\frac{\partial}{\partial Q_{j}} u_{k}(Q)+\frac{\partial}{\partial Q_{k}} u_{j}(Q)\right) N_{Q}^{j}=a_{k}(Q), \quad k=1, \ldots, n
$$


where the $a_{k}$ are unit atoms (take $0 \in \partial D, \operatorname{supp} a_{k}=\{Q:|Q|<1\}$, $\left\|a_{k}\right\|_{L^{\infty}(\partial D)} \leq 1$, and

$$
\int_{\partial D} a_{k}(Q) d Q=0
$$

Problem: Obtain an estimate when $n \geq 4$,

$$
\sum_{k=1}^{n} \int_{\partial D}\left(\nabla u_{k}\right)^{*} d Q \leq C,
$$

where $C$ is independent of $\vec{a}$, and ( $)^{*}$ is the nontangential maximal function. [Note: This estimate is true for $n=2,3$; proofs by Dahlberg, Kenig.]

17. (G. Verchota). Let $\Delta^{2} u=0$ in $D \subset \mathbf{R}^{n}$. On the boundary let $u(Q)=0$ and let $\partial u(Q) / \partial N \in L^{p}(\partial D)$, where $p^{-1}=2^{-1}-(n-1)^{-1}$. Problem: Obtain the estimate when $n \geq 4$

$$
\int_{\partial D}(\nabla u)^{* p} d Q \leq C \int_{\partial D}\left|\frac{\partial u}{\partial N}\right|^{p} d Q .
$$

[Note: For $n=4,5$, this is the best one can expect (V. Pipher).]

18. (M. Wilson). Let $S(f)$ be the dyadic square function and let $f^{*}$ be the dyadic maximal function on $\mathbf{R}^{d}$. Let $M^{k}$ denote the $k$-fold application of the Hardy-Littlewood maximal operator (e.g., $\left.M^{2} V=M(M V)\right)$. The following inequalities are know to be true:

$$
\int\left|f^{*}\right|^{p} V \leq C(p, d) \int S^{p}(f) M^{[p / 2+1]} V
$$

for every $f \in C_{0}^{\infty}$ and $V \geq 0$ in $L_{\text {loc }}^{1}$,

$$
\int S^{p}(f) V \leq C(p, d) \int|f|^{p} M V, \quad 1<p \leq 2 .
$$

Is the following inequality true:

$$
\int S^{p}(f) V \leq C(p, d) \int|f|^{p} M^{k(p)} V, \quad \text { as } p \rightarrow \infty,
$$


with $k(p) \sim p / 2$ ? In particular, with $k(p)=] p / 2[$, where $] x[\equiv-[-x]$ ?

19. (M. Wilson). Let $\alpha>0$. Does there exist a $C_{\alpha}<\infty$ so that the following is true: if $V$ is a nonnegative weight and $\Re$ a family of double dyadic rectangles such that

$$
\frac{1}{|R|} \int_{R} V \leq 1, \quad \text { for all } R \in \Re,
$$

then given

$$
\begin{gathered}
g=\left(\sum_{R \in \Re} \lambda_{R} \chi_{R}\right)^{\alpha}, \quad \lambda_{R} \geq 0, \\
\int g V \leq C_{\alpha} \int g ?
\end{gathered}
$$

Note: Answer is yes with $C_{\alpha} \equiv 1$ if $\Re$ is a family of dyadic cubes.

\section{REFERENCES}

1. J. Garnett, Bounded analytic functions, Academic Press, New York, 1981.

2. J.-L. Journé, Calderón-Zygmund operators, pseudo-differential operators, and the Cauchy integral of Calderón, Lecture Notes in Math. 994 (1983),

Department of Mathematics, University of Montana, Missoula, MT 59812 